\title{
Performance Analysis of SIFT Feature Extraction Algorithm in Application to Registration of SAR Image
}

\author{
Yan Li SUN, Jie WANG
}

Department of basic experiment, Naval Aeronautical and Astronautical University, Yantai264000,China

\begin{abstract}
Exactly extracting the stable feature of high resolution SAR image as well as matching it are two critical steps for the Antomatic regiestation systems. It is suggested that the Scale Invariant Feature Transform (SIFT) algorithm can be applied in the optical image registration systems and four representative experiments were performed to test its validity. It is found that SIFT can accurately register the high resolution SAR images than the traditional Harris in applicability and precision.
\end{abstract}

Keywords: SAR Imagery, SIFT, Harris, Automatic Registration.

\section{Introduction}

Image registration $[1,2]$ is the process of aligned two or more images in space from different time, different perspectives and different sensors, and it is a key step of multi-source image fusion or change detection. It can be divided into semi-automatic and automatic image registration according to people's participation. Semi-automatic registration extract feature based on human-computer interaction, and then match the feature, transformation and re-sampling using the computer. Automatic registration accomplishes registration by computer directly without consumer's participation.

Automatic registration of SAR image especially High-resolution SAR image has not been well solved for a long time, sub-pixel registration accuracy is often required to achieve in practical applications. It put forward higher requirements to feature extraction and feature matching algorithm. The method Based on regional correlation applies more in optical remote sensing image registration. However, it is difficult to apply in the high-resolution SAR images, because it is more sensitive to image gray, rotation and objectives' increase or decrease, and its high complexity computation of using all points of regional information. The key of another registration method based feature is to establish the key points, lines or other geometric corresponding relationship. As the SAR image of special imaging modalities and imaging mechanism, in particular a large number of speckle noise, some feature extraction algorithm take good performance in optical image registration but failed in SAR image. Some more robust feature extraction algorithm need to be introduced.

This article discusses the application of SIFT feature extraction algorithm in high resolution SAR image registration. SIFT algorithm is aimed to the SAR image's in four representative designed experiments. It is compared with traditional Harris corner point in running time, the correct rate of the matching and the accuracy of the key points . According to qualitative and quantitative analysis, SIFT can accurately register the high resolution SAR images were acquired in the same ascending orbit, and it is better than 
Harris in applicability and precision, and analysis limitations of the two algorithms.

\section{Registration of SAR Image based on feature matching}

First extract feature point by feature extraction operators in the reference image and the registration image, and then match the feature point using a similarity measure, at last use two-way relevance method and the RANSAC to remove external point to improve the registration accuracy. As shown in Fig. 1.

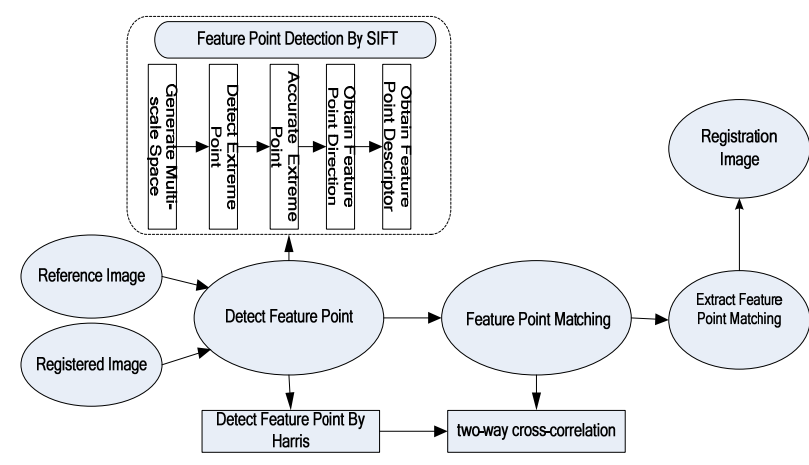

Figure 1. Flow chart of SAR Image Registration based on feature matching

\subsection{Image registration based on Harris}

Harris operator is Proposed by C.Harris[6] that based on extraction operator of signal point feature. Even if there is image rotation, gray change, noise and viewpoint change and so on in the optical image, Harris is also the most stable operator of point feature extraction operator. $\mathrm{Fu}$ Kun[7]register SAR image by hand first then by Harris operator and at last using small panel to make registration accuracy better than 1 pixel.

This operator inspirited by the autocorrelation function of the signal processing, given the matrix $\mathrm{M}$ associated with the autocorrelation function. Eigen values of matrix $M$ is first-order curvature of the autocorrelation function, the point is the feature points if the two curvature values are high. The operator expression of Harris:

$$
\begin{aligned}
& R=\operatorname{det}(M)-k t^{2}(M) \\
& M(x, y)=\left[\begin{array}{ll}
I_{u}^{2}(x, y) & I_{u v}(x, y) \\
I_{u v}(x, y) & I_{v}^{2}(x, y)
\end{array}\right]
\end{aligned}
$$

where $I_{u}(x, y)$ and $I v(x, y)$ are the partial derivative of the pixel gray value of point ( $\mathrm{x}$, $\mathrm{y})$ in the direction of $\mathrm{u}$ and $\mathrm{v}, \operatorname{Iv} u(x, y)$ is mixed partial derivative of second order; det is the determinant of matrix, tr is the trace of matrix, $\mathrm{k}$ is determined by experience, usually take 0.04 .If $\mathrm{R}$ exceeds a certain threshold, the point is considered the corner point. Realize matching by normalized cross correlation of the center intensity of the local image after the extraction of same feature point.

\subsection{The registration based on SIFT}

SIFT is a algorithm that can extract local feature, the core idea is to find extreme points in scale space, extract invariant when location, scale, rotation, illumination changed. The algorithm is proposed by David Lowe [3] [4] in 1999 and to be completed in 2004. The algorithm is robust with scale, rotation, brightness, affine, and so is invariant noise, increase or decrease in the target and blocks. Mikolajczyk [5] do experiment and compare the performance with most representative descriptors (10 descriptors such as SIFT, matrix invariants, cross correlation and so on) under different scenes. The results show that, the performance of SIFT descriptor is best. SIFT method includes three steps: detection of extreme point in scale space, the formation of feature point descriptor and feature point matching.

\subsubsection{Detection Extreme point in scale space}

SIFT algorithm usually form a pyramid by down-sampling the original image, build filter images group by each image convolute with Gaussian kernel convolution of different scales. To detect the stability of the feature points, minus each layer image of each group is required to form a difference of Gaussian scale space (DOG). Each pixel compared with the adjacent eight pixels of the same scale and around the scale $9 \times 2$ pixels surrounding neighborhood to ensure that local extremum can be detected in the scale of space and two-dimensional image space. Sub-pixel level positioning with quadratic functions, while removing some of the edge point of instability. 


\subsubsection{The formation of feature points descriptor}

Determine the main direction of feature points through the gradient direction distribution characteristic of neighborhood pixel, the $16^{*} 16$ window of the center of feature points is divided into $4 * 4$ sub-windows, each sub-window calculate gradient direction histogram of 8 directions by Gaussian-weighted, form the $4 * 4 * 8$ vector that is the feature point descriptor.

\subsubsection{Feature points matching}

Take a feature point of reference image, find out the feature points in the registration image that Euclidean distance between them is smallest and second smallest. If the value that divide the nearest distance by second nearest distance is less than a certain threshold that is the right match. In order to ensure the accuracy of matching points, two-way matching is used.

\subsection{Purifying matching point by RANSAC}

Although the right rate of matching point is high by using two-way nearest neighbor method in SIFT algorithm, but then the mismatch is inevitable. The basic idea of RANSAC algorithm is: select two points randomly, determine a straight line by two points, the points within a certain distance in this line called the interior point, the rest called external point. Select repeat times randomly, the straight line with the most interior points is recognized as a sample point set and to exclude external point.

\subsection{Construction of polynomial realize the registration}

Model parameters of spatial transformation can be get by extracting feature points using polynomial equation of least squares:

$$
\begin{aligned}
& X c=a 0+a 1 x r+a 2 y r+a 3 x r y r+a 4 x 2 r+a 5 y 2 r \\
& Y c=b 0+b 1 x r+b 2 y r+b 3 x r y r+b 4 x 2 r+b 5 y 2 r
\end{aligned}
$$

The coordinate of registration points is expressed as $(\mathrm{Xc} ; \mathrm{Yc})$, and the coordinate of reference points is expressed as $(\mathrm{Xc} ; \mathrm{Yc})$. Images will be transformed in space and registered using equation (1).

\section{Experiment and Analysis}

In order to analysis and compare application performance of two feature extraction operators comprehensive, we selected four pairs of typical SAR images, The first major is the change in brightness and rotation angle and a small number of target changes (Figure 2 ). The second not only brightness changes greatly screen on different directions but also a large number of targets changes (Figure 3). the main difference of the third is resolution (Figure 4). The fourth pair is obtained by exactly the opposite direction of flight (Figure 5).

The accuracy and time of SIFT and Harris extract feature points is shown in Table I . Precision of feature points in the first pair SAR images that detected by two method is compared particularly in Table II. It can be seen, the scope of classic Harris corner detection algorithm is very narrow in SAR images, and time-consuming is long.

In the first pair images Harris can extract enough feature points, but usually use maximum mutual correlation as similarity measure to find matching points, and it is sensitive with the rotation angle, resulting many errors in matching point pairs. Complete the registration after using RANSAC to remove error matching point pairs but the average accuracy only reached 2.12 pixels. The SIFT not only can extract enough feature points but also its' correct matching rate can reach $100 \%$ by the method of two-way the largest neighboring matching (Figure 6). Accuracy can reach 0.52 pixels on average after the image registration. In the second pair images, only a small amount of feature point is extracted by SIFT because of big perspective (Figure 3), However, registration accuracy can still achieve sub-pixel, the average deviation in horizontal direction is 0.928 pixels, in vertical direction is 0.7 pixels. But Harris can not extract the correct feature points that can be used to match, particularly in the target changed area, so indicates that SIFT is stable with the increase or reduction of the target. In the third pair Harris can not extract the correct feature points because it is not invariant on the scale changed. But SIFT not only extract a large number of feature points but also 
matching feature points absolutely right (Figure 4), so confirms its scale invariant.

There is difference between SAR image and ordinary optical image. There will be a completely different texture features of SAR image obtained by entirely different angle, so can not get satisfactory results using SIFT. In

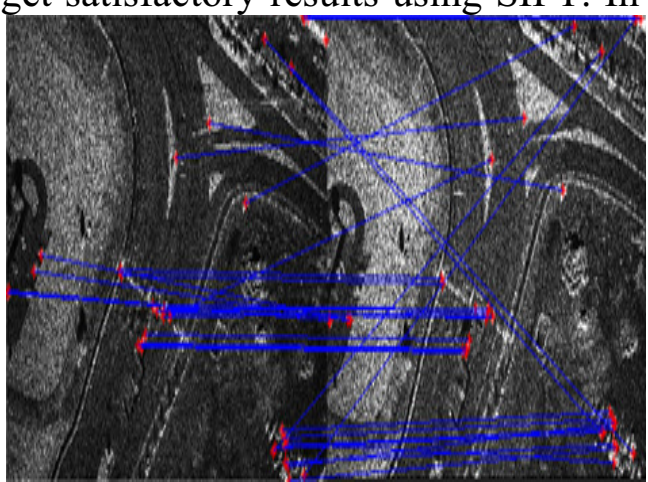

Figure 2. Matching points on the first image by Harris and SIFT (Image size is 2510*1638)
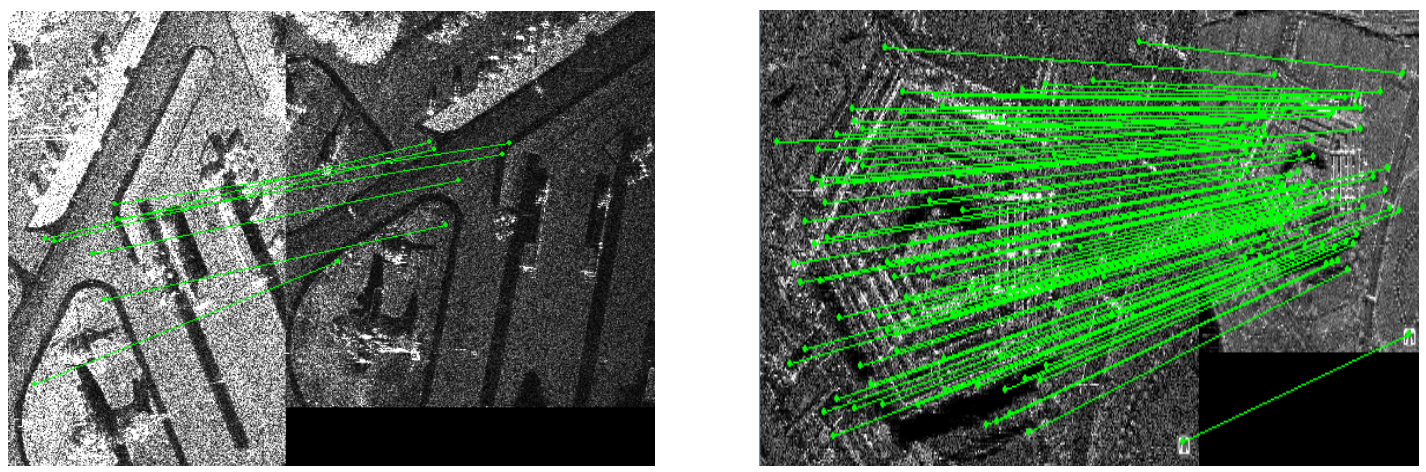

Figure 3. Matching points on second image by SIFT $\left(750^{* 1150} 1000 * 1000\right)$ Figure4.Matching points on third image by SIFT $(1200 * 800600 * 600)$

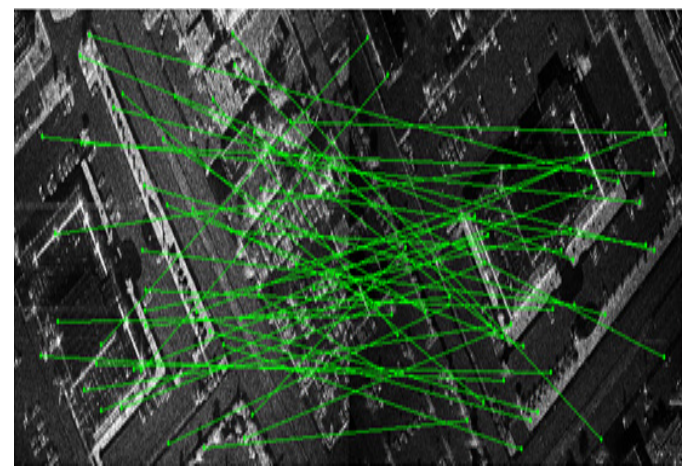

Figure 5. Matching points on the fourth image by SIFT (Image size is $2510 * 1638$ )

TABLE 1. Compared SIFT algorithm with the Harris

\begin{tabular}{|l|l|l|l|l|l|l|}
\hline \multirow{2}{*}{$\begin{array}{l}\text { Im } \\
\text { age }\end{array}$} & \multicolumn{3}{|l|}{ SIFT } & \multicolumn{2}{l|}{ Harris } \\
\cline { 2 - 7 } & $\begin{array}{l}\text { Matching } \\
\text { Points }\end{array}$ & $\begin{array}{c}\text { Accuracy } \\
\text { Rate }\end{array}$ & Time & $\begin{array}{c}\text { Matchin } \\
\text { g Points }\end{array}$ & $\begin{array}{l}\text { Accuracy } \\
\text { Rate }\end{array}$ & Time \\
\hline 1 & 150 pairs & $100 \%$ & $50.328 \mathrm{~s}$ & 32 pairs & $53.1 \%$ & $629.71 \mathrm{~s}$ \\
\hline 2 & 7 pairs & $100 \%$ & $10.207 \mathrm{~s}$ & 0 pair & $0 \%$ & $935.12 \mathrm{~s}$ \\
\hline 3 & 63 pairs & $100 \%$ & $8.251 \mathrm{~s}$ & 34 pairs & $0 \%$ & $516.72 \mathrm{~s}$ \\
\hline 4 & 17 pairs & $17.6 \%$ & $53.178 \mathrm{~s}$ & 0 pair & $0 \%$ & $985.62 \mathrm{~s}$ \\
\hline
\end{tabular}


TABLE 2. the accuracy of feature point extraction compared SIFT with Harris in the first pair of SAR images

\begin{tabular}{|c|c|l|l|l|l|}
\hline $\begin{array}{c}\text { Algorit } \\
\text { hm }\end{array}$ & NO & $\begin{array}{l}\text { Coordinate Of } \\
\text { Reference } \\
\text { Image }\end{array}$ & $\begin{array}{l}\text { Registration } \\
\text { value }\end{array}$ & True value & $\begin{array}{l}\text { Horizontal } \\
\text { Vertical } \\
\text { Deviation }\end{array}$ \\
\hline \multirow{5}{*}{ SIFT } & 1 & $(1922.6,924)$ & $(1926,886.19)$ & $(1925.8,885.04)$ & $(-0.2,-1.15)$ \\
\cline { 2 - 7 } & 2 & $(769.32,1534.3)$ & $(840.68,1579.3)$ & $(840.4,1579.4)$ & $(-0.28,0.1)$ \\
\cline { 2 - 7 } & 3 & $(1652,1056.2)$ & $(1671,1035.4)$ & $(1670.6,1036.9)$ & $(-0.4,0.1)$ \\
\cline { 2 - 7 } & 5 & $(2152.4,1453.2)$ & $(2208,1396.8)$ & $(2208.6,1396.3)$ & $(0.6,-0.5)$ \\
\hline \multirow{5}{*}{ Harris } & 1 & $(2118.5,1030.6)$ & $(1216.8,1043.1)$ & $(1216.7,1044.9)$ & $(-0.1,1.8)$ \\
\cline { 2 - 7 } & 2 & $(2152.4,1453.2)$ & $(2176.7,2208)$ & $(2179.2,2211.1)$ & $(2.5,3.1)$ \\
\cline { 2 - 7 } & 3 & $(881.91,938.09)$ & $(893.67,9796.8)$ & $(1403.8,1394.2)$ & $(1.3,-2.6)$ \\
\cline { 2 - 7 } & 4 & $(1633.4,1210.6)$ & $(1665.8,1402.5)$ & $(1695.6,973.1)$ & $(1.93,-2.5)$ \\
\cline { 2 - 7 } & 5 & $(1140.3,970.14)$ & $(1155.7,991.04)$ & $(1152.6,993.2)$ & $(0.4,-1.3)$ \\
\hline
\end{tabular}

\section{Conclusion}

In this paper, the SIFT algorithm is proposed and compared with Harris in high resolution SAR image registration. The result of experiments shows that SIFT can accurately register the high resolution SAR images that acquired in the same ascending orbit with different translation 、 rotation 、 scaling 、 brightness 、 the increase or reduction of the targets and view angle changes. The precision of registration can reach sub-pixel. The Harris can not extract the correct feature points in scaling or perspective changing image, and is also sensitive to increase or reduction of the targets. Thus SIFT is better than Harris in applicability and precision. SIFT can not extract invariant feature in SAR images that acquired in the different ascending orbit. How to solve this type of image registration issues will be the focus of future research.

\section{References}

[1] L G Brown. A survey of image registration techniques[J]. ACM Computing Surveys (S0360-0300),1992,24(4):326-376.

[2] B Zitová, J Flusser. Image registration methods: a survey [J].Image and Vision Computing(S0262-8856),2003,21(11):977-1000.

[3] Lowe D G. Object recognition from local scale-invariant features[A].International Conference on Computer Vision[C], Cor-fu, Greece, Sep 1999: 1150 1157.

[4] Lowe D G. Distinctive image features from scale-invariant key-points[J].International Journal of Computer V ision,2004,60(2): 91 110.

[5] Mikolajczyk K, Schmid C. A Performance Evaluation of Local Descriptors[ J].IEEE Trans. Pattern Analysis and Machine Intelligence,2005,27(10): 1615-1630.

[6] HarrisC G, StephensM J. A Combined Corner and EdgeDetector[A]. Proceedings Fourth Alvey Vision Conference[C].Manches-ter, 1988

[7] Kun Fu,Hongjian You.Precise research about matching technology of multi-temporal spaceborne SAR images[J]. Remote Sensing Technology and Application,2007,10(5):37-05 